\title{
Practice and experience of task management of university students: Case of University of Tsukuba, $\operatorname{Japan}^{1}$
}

\author{
Ryoko Fukuzawa $^{\mathrm{a}}$, Hideo Joho ${ }^{\mathrm{b}, *}$ and Tetsuya Maeshiro ${ }^{\mathrm{b}}$ \\ ${ }^{a}$ Graduate School of Library, Information and Media Studies, University of Tsukuba, Tsukuba, Ibaraki, \\ Japan \\ ${ }^{\mathrm{b}}$ Research Center for Knowledge Communities, Faculty of Library, Information and Media Science, \\ University of Tsukuba, Tsukuba, Ibaraki, Japan
}

\begin{abstract}
This paper reports the results of a survey that investigated the practice and experience of task management of university students. A total of 202 tasks identified by 24 university students were analyzed. The results suggest that participants had a reasonable sense of priority of tasks, that they tend to perceive a task as a big chunk, not a series of small chunks, that estimated time can be a good indicator of task completion, that time management and loss of coordination are the major factors for uncompleted or non-started tasks, and finally that their notion of task grouping was dominated by task themes or topics.
\end{abstract}

Keywords: Survey, task management, higher education

\section{Introduction}

It is common for us to be involved in multiple tasks in our daily life, and task management is a key issue to maximize the productivity and sustainability of our work. Task management has been studied in several domains. For example, Czerwinski et al. [4] investigated the practice of task switching and intervention using a diary-based approach, and reported how task productivity can be degraded by several forms of intervention that prevent people from performing the task that they ought to be doing. Levy et al. [7], on the other hand, examined the effect of meditation on task management and performance. Coviello et al. [3] proposed a formal model of product function where multiple tasks are involved within a given resource. In information seeking and retrieval, Lin and Belkin [8] proposed taxonomy of tasks in the context of information seeking. Agichtein et al. [1] examined how search tasks were interrupted and resumed. On the other hand, multitasking has been studied in

\footnotetext{
${ }^{1}$ This is a revision of the paper originally published at A-LIEP 2013.

* Corresponding author: Hideo Joho, Research Center for Knowledge Communities, Faculty of Library, Information and Media Science, University of Tsukuba, 1-2 Kasuga, Tsukuba, Ibaraki 305-8550, Japan. Tel.: +81 29859 1337; Fax: +81 29859 1093; E-mail: hideo@slis.tsukuba.ac.jp.
} 
the context of the Web as well as libraries [10[11]. However, limited attention was paid to the progress or performance of multiple tasks.

It is evident that task management is a crucial aspect for professionals when handling multiple tasks. For example, Bellotti et al. [2] investigated seven professionals for their task management behavior and tools used, and identified several task management tactics. They also proposed a design of 'to-do' lists based on their findings. However, a recent study shows that task management can be an important skill for those in higher education, such as university students. For example, Junco and Cotton [6] investigated the effect of instant messenger (IM) use on students' academic performance, and observed a negative effect of IM on performance. Furthermore, O'Brien and Toms [9] found that multitasking could degrade the level of engagement with tasks. These findings suggest that improving task management skills is just as important for students as it is for professionals.

Given that researchers, practitioners, and educators in Library and Information Science (LIS) have been striving to organize and deliver a range of resources recorded in various formats, the authors believe that there is scope in LIS services to support people in organizing and executing their tasks according to a plan. In particular, university libraries have been among the most important spaces for students to perform various learning processes. However, we have limited understanding of the practice and experience of students' task management in higher education. One exception is Haraty et al. [5] who conducted semi-structured interviews to investigate how faculty and students manage tasks. Their study suggests that there are different styles in task management and how tools are used. However, their study did not look in detail at the effect of individual styles on task progress.

Based on such observations, this study aims to gain an insight into the practice and experience of task management by university students and explore potential ways to support them. More specifically, our survey investigated the following aspects of task management in higher education.

- What do university students perceive as a task?

- To what extent do university students perform the perceived tasks?

- To what extent are university students aware of the time needed for tasks?

- To what extent do university students prioritize tasks?

- How do university students group tasks?

It should be emphasized that this survey did not look at the performance of individual tasks. In other words, although we analyzed whether or not a task had been completed, investigating whether or not such a task was successful was beyond the scope of this study.

The rest of the paper consists of the following: Section 2 describes the survey designed to answer the research questions given above; Section 3 presents the results of data analysis; Section 4 summarizes the major findings of the survey and discusses their implications for the design of task management support. Finally, Section 5 concludes the paper with future work. 


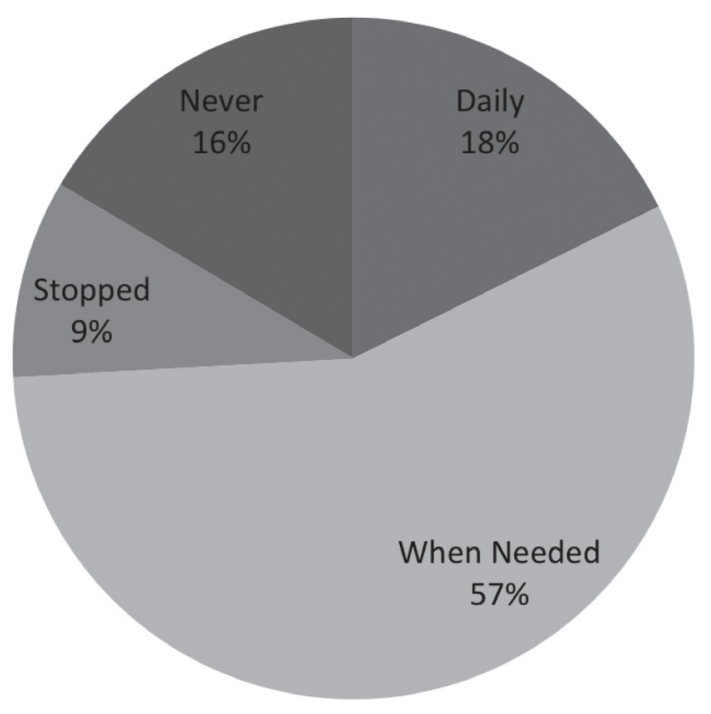

Fig. 1. Daily practice of task management.

\section{Methodology}

This section describes the method used to survey the practice and experience of task management by university students in this study.

\subsection{Terminology and scope}

Although the term "task management" is used throughout this paper, we used the term "to-do list" with the following description in the survey to ensure that participants understood the aim and scope of the study.

A to-do list is a list of things that a person needs to complete by a certain date. It does not only include work-related tasks; any tasks can be considered, such as hobbies, shopping, social activities, and so on.

\subsection{Short survey of needs}

Before investigating the perception and practice of university students' task management, we performed a short survey of the needs of task management. A questionnaire which asked two questions about their current task management practice was distributed to a class of third-year students of Library and Information Science at our institution. As a result, we had 85 valid responses from the students in the class.

The first question (see Fig. 1) asked about their experience of task management, and $17.6 \%$ answered that they do task management on a daily basis, while $56.5 \%$ answered that they do it in on a need-to-do basis, and $25.9 \%$ answered either that 


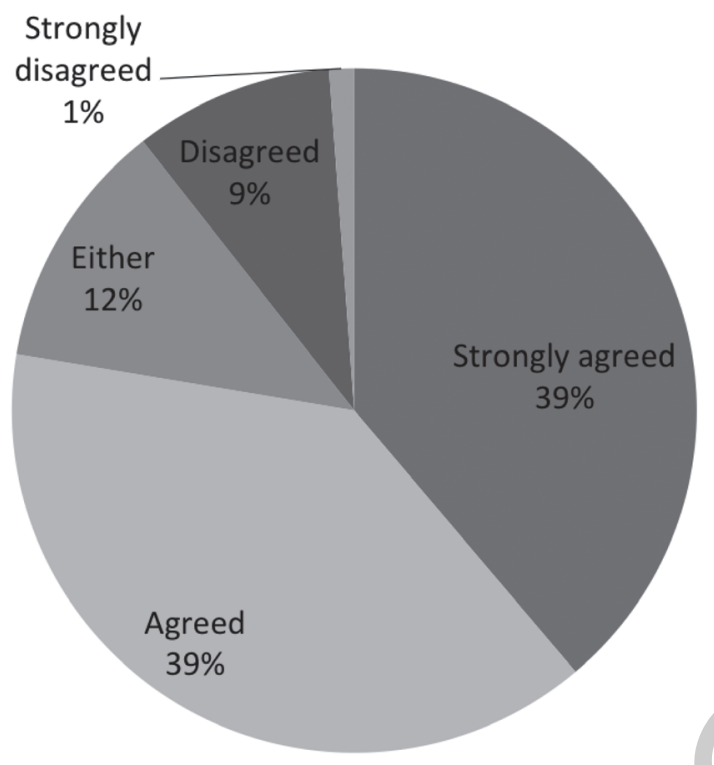

Fig. 2. Need to improve task management skills.

they used to do it, but do not do it anymore, or that they have never done task management. The second question (see Fig. 2) asked about their perception of the need to improve their task management skills. Participants were asked to indicate their level of agreement to the following statement "I need to improve my task management skills". To this, 38.8\% answered "strongly agree", 38.8\% answered "agree", $11.8 \%$ answered "either" (i.e., neutral), and 10.6\% answered either "disagree" or "strongly disagree".

The findings of this short survey reinforced the need to understand the task management skills of university students and support our motivation to look into these issues in this paper. The next sections will describe our investigation to clarify their perceptions and practice of task management in more detail. Note that the participants in the short survey and the main survey were different.

\subsection{Survey forms}

The survey forms devised for this study consisted of two parts. Participants were asked to fill in the first part of the form when they participated in the study (called "first week" in the paper), and one week later, they were asked to fill in another questionnaire (called "second week" in the paper).

\subsubsection{First week}

The first week of the survey had two pages. The first page asked for participants' demographic information and previous experience of task management. This page 


\begin{tabular}{|l|l|l|l|}
\hline ID & Task & $\begin{array}{l}\text { Estimated } \\
\text { Time }\end{array}$ & Priority \\
\hline 1 & Write the section of Experiment I & $10 \mathrm{H} 0 \mathrm{M}$ & ( $/$ / M / L \\
\hline 2 & Buy Christmas gifts & $1 \mathrm{H} 30 \mathrm{M}$ & $\mathrm{H} / \mathrm{M} / \mathrm{L}$ \\
\hline 3 & $\cdots$ & & \\
\hline
\end{tabular}

Fig. 3. Form to describe perceived tasks.

\begin{tabular}{|c|l|c|}
\hline ID & Progress status & Actual Time \\
\hline 1 & Completed, uncompleted, not started & H M \\
\hline 2 & Completed, uncompleted, not started & H M \\
\hline 3 & $\ldots$ & \\
\hline
\end{tabular}

Fig. 4. Form to report the progress of tasks and actual time taken.

also explained what we meant by a task and task management as discussed in Section 2.1. Apart from the explanation of tasks, we also explicitly asked participants to write down only those parts of a task that needed to be done within the next week, even if those tasks might be part of continuous tasks.

The second page asked participants to think of and write down all tasks that needed to be done within the following week. We also asked them to estimate the time needed to complete individual tasks and their priority level (High, Medium, and Low). The sample form of the task list is shown in Fig. 3.

\subsubsection{Second week}

Participants were asked to come back a week later and report the progress of each of the tasks they had identified in the first week. If any of those tasks had not progressed as planned in the first week they were asked to describe the reasons why. More specifically, we asked them to indicate the following aspects of their task management experience.

1. Progress and actual time taken for individual tasks

2. Reasons for those tasks that were not completed

3. How tasks were grouped

4. Any opinions about their task management practice and experience

5. Any feedback on the survey

The sample form used in the second week is shown in Fig. 4. Three categories of progress status were defined as completed, uncompleted, and not started. A completed task was any task that was completed within the last week as planned. An 
uncompleted task was any task that was started but not completed as planned. A not started task was any task that had not even been started. When participants marked a task either completed or uncompleted, they were asked to indicate the amount of time spent on the task. Participants filled in the second week's survey while looking at their first week's survey.

After evaluating all tasks, participants were asked to categorize the tasks into groups. We did not give any instruction on how tasks should be categorized, which allowed us to study the students' perception of task groups. They were given another form to write down a set of task IDs and a label for the group.

A pilot study was carried out with five participants to ensure that the survey protocol, clarity of survey instructions and forms, and time allocation were appropriate. Most of the problems found during this pilot study were minor, however, we learnt that we should clarify that participants could include a task that was continuing for a longer period, but only the part that needed to be done in the following week.

\subsection{Protocols}

A survey on individual participants was carried out in the following manner.

1. 1st week

Ask them to read the information sheet that describes the aim of the survey

Ask them to sign the consent form if they agreed to participate

Ask them to fill in the first survey form.

2. 2nd Week

Ask them to fill in the second survey form

A reward was given to them and they were asked to sign a receipt

Participants were rewarded with 500 JPY (approx. \$5) on the completion of the second week; they were told that they would receive this in the information sheet.

\subsection{Participants}

This survey was carried out in the University of Tsukuba, Japan. A call for participation was published on the lab's website, and distributed via several mailing lists in the University. Participants were accepted on a first come first served basis. A total of 24 students (18 females and 6 males) were recruited for the study. Of those, 13 were undergraduate students and 11 were postgraduate (Masters and $\mathrm{PhD}$ ) students. Their academic background varied from Library and Information Science (10) to Computer Science and Engineering (4), Life Science (4), Education (2), Arts (1), Literature (1), Sociology (1), and Psychology (1).

\subsection{Period of survey}

The survey took place between December 2012 and January 2013. This is a busy time of year for Japanese students due to the holiday season and graduation report 
deadlines. We intentionally selected this period so that many tasks could be elicited from the students, and their level of engagement (i.e., seriousness) can be greater at this time than during other seasons. The implication of this design will be discussed later in the paper.

\section{Results}

Twenty-four participants reported a total of 202 tasks as something they needed to complete within a week from the day they filled in the first part of the survey. The mean value was 8.42 (SD: 3.69). Of those, 85 were marked as high priority, 73 as medium priority, and 44 as low priority by participants. This section presents the results of analysis based on those reported 202 tasks.

\subsection{Daily practice of task management}

At the beginning of the survey sheet for the first week, we asked participants how they performed task management. The result shows that four do task management on a daily basis, 18 do it on a need-to-do basis, and two have never done task management. Therefore, although most participants had some experience of task management, only $17 \%$ of students do it as a daily practice.

The tools they used for task management (multiple choice) were pen and paper (10), analog diary and calendar (11), smart phone apps (5), and PC (5). Many reported that they used multiple tools for task management.

\subsection{Perceived objectives}

First, we broadly categorized the objectives of reported tasks and the result is shown in Fig. 5. As can be seen, $40 \%$ of tasks were related to study and research, followed by housekeeping (16\%), socializing (10\%), and job hunting (9\%). These results suggest that the survey was successful in capturing non-work related tasks in the study.

When we took a closer look at individual tasks, we noticed a big difference in how students perceived a task. In particular, some tasks were described as one big chunk of work such as "completing the graduate project report", while other tasks were divided into smaller chunks such as "writing an introduction to the report" and "analyzing the experimental results". However, measuring the size of tasks in an objective manner is not trivial. Nevertheless, it seems reasonable to assume that some tasks were perceived as a big chunk while others were seen as a series of smaller chunks. This seems to be related to their estimation of task completion time. While it was estimated that half of the reported tasks could be completed in less than 90 minutes, the other half ranged from 120 minutes to 1,200 minutes. Clearly, in the latter case students were not able to divide a big task into smaller chunks. 


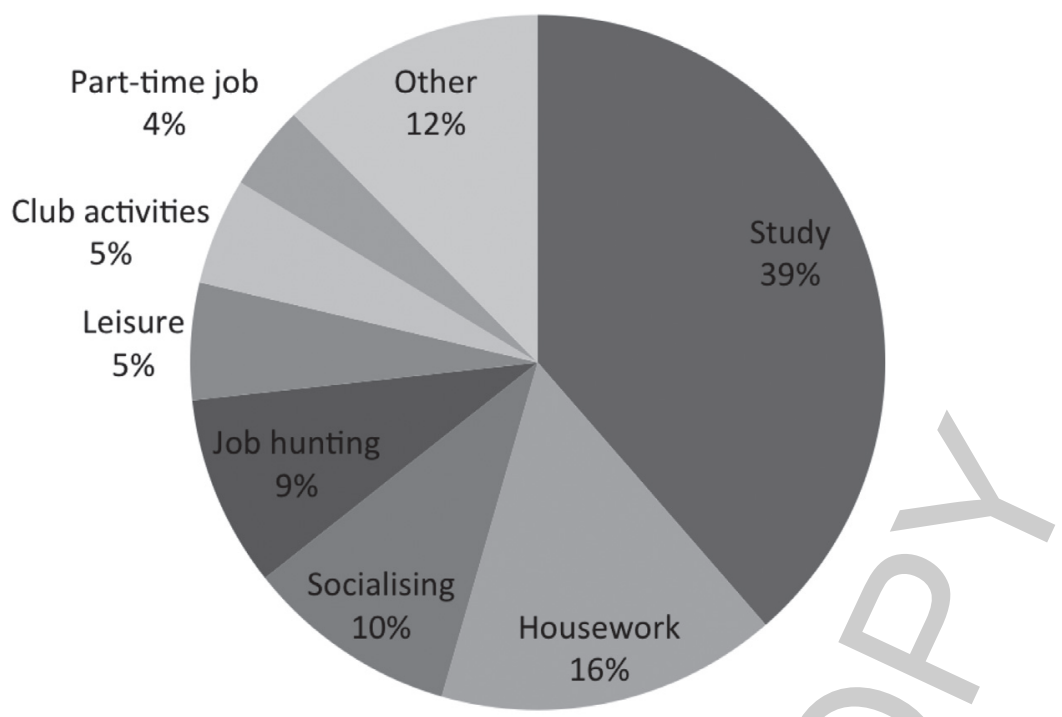

Fig. 5. Perceived objectives of tasks.

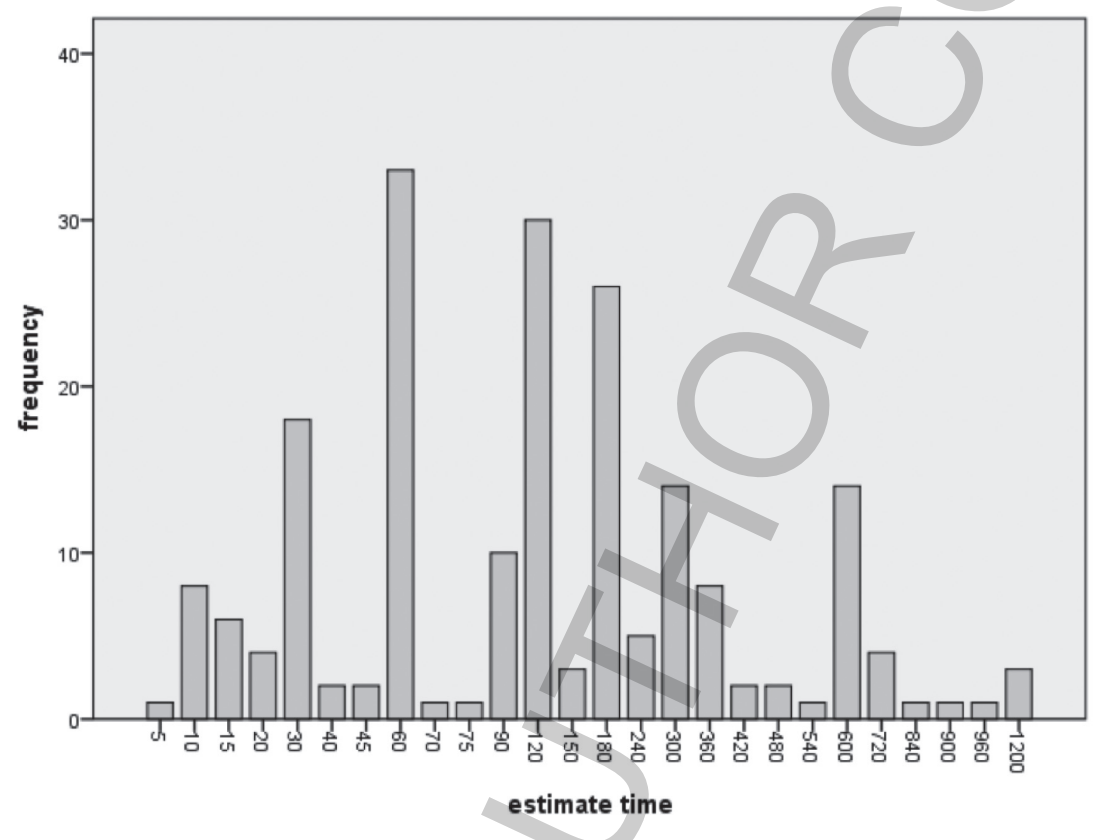

Fig. 6. Distribution of estimated task completion time. 


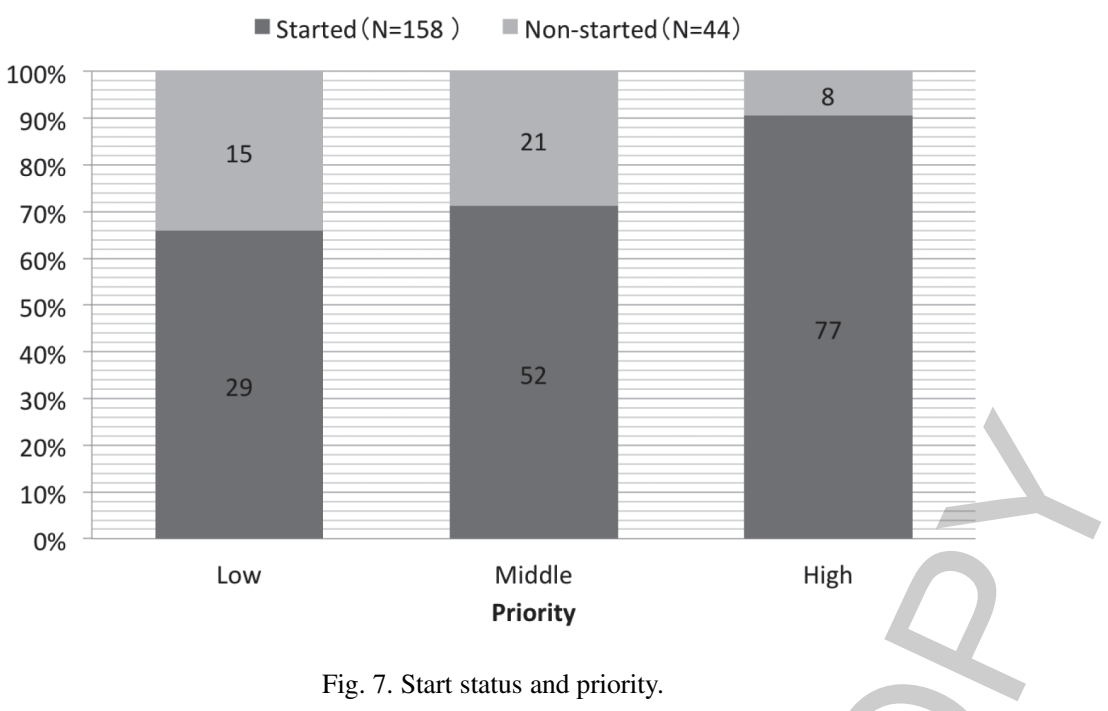

In this paper, we assume that the size of a task can be inferred from the participant's estimate of how long it will take to complete. For reference, the mean estimated task completion time was 203.6 minutes (SD: 232.5) and the median was 120 minutes (IQR: 240). The histogram of estimated task completion time is shown in Fig. 6. More details will be discussed in the following sections.

\subsection{Started and non-started tasks}

In the second week of the survey, we asked participants to code the status of all tasks. One category of status was whether or not a task had been started. We called those tasks that had been started "started task", and those which had not "non-started task" in this paper. The counting of participants' coding shows that 158 tasks (78.2\%) were started within a week and 44 tasks $(21.8 \%)$ were not. We performed a series of analyses to understand the characteristics of started and non-started tasks as follows.

First, we looked at the relationship between the start status and priority, and the results are shown in Fig. 7. As can be seen, most high priority tasks were started, and the proportion of started tasks decreased as the priority got lower. This suggests that students have a sense of priority and managed to start those tasks with a high priority.

Second, we looked at the relationship between the start status and the estimated length of task completion time. As discussed earlier, we assumed that the size of tasks could be inferred from the estimated task completion time. Therefore, we are interested in the relationship between the task size and the start status. The results are shown in Fig. 8, where the x-axis represents estimated task completion time in minutes. 


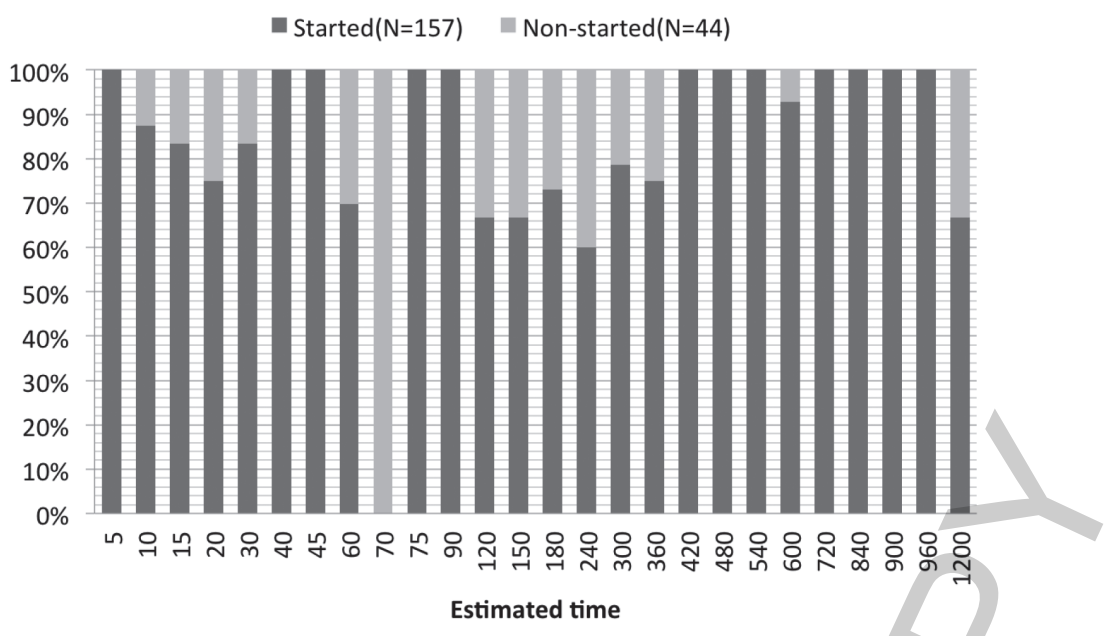

Fig. 8. Start status and estimated time.

As can be seen, we did not find a clear pattern in the distribution of non-started tasks over the estimated task completion time. This suggests that a student's perception of a task as big or small does not necessarily indicate whether or not they will engage with the tasks.

\subsection{Completed and uncompleted tasks}

The previous section looked at started and non-started tasks. Of those 158 started tasks, there are 113 tasks (55.9\%) that are coded as completed within a week as planned, and 45 tasks (22.3\%) that are coded as uncompleted. This section presents a series of analyses to understand the characteristics of completed and uncompleted tasks.

The first analysis, as with the started and non-started tasks, looked at the relationship between completion status and priority. The results are shown in Fig. 9. As can be seen, the proportion of completed tasks did not vary across the level of priority. Therefore, a student's prioritizing of a task does not seem to correlate with the probability of task completion.

For those tasks that were started, we asked participants to report how long they spent on the task between the first and second week. As we all know from experience, some tasks take more time than we had expected, while others take less. In other words, there is often a gap between estimated time and actual time spent on a task. The second analysis looked at the relationship between the completion status and the size of gap. The gap was calculated as follows.

Gap $=($ Actual time - Estimated time $) /$ Estimated Time $\times 100$ 
Table 1

Gap between estimated and actual time of completed tasks

\begin{tabular}{cccc}
\hline & Frequency $(N=112)$ & Median $(\mathrm{IQR})$ of gap & Median (IQR) of estimated time \\
\hline Gap $>0$ & $25(22.32 \%)$ & $100(100)$ & $90(97.5)$ \\
Gap $=0$ & $43(38.39 \%)$ & & $120(120)$ \\
Gap $<0$ & $44(39.28 \%)$ & $-50(16.67)$ & $90(150)$ \\
\hline
\end{tabular}

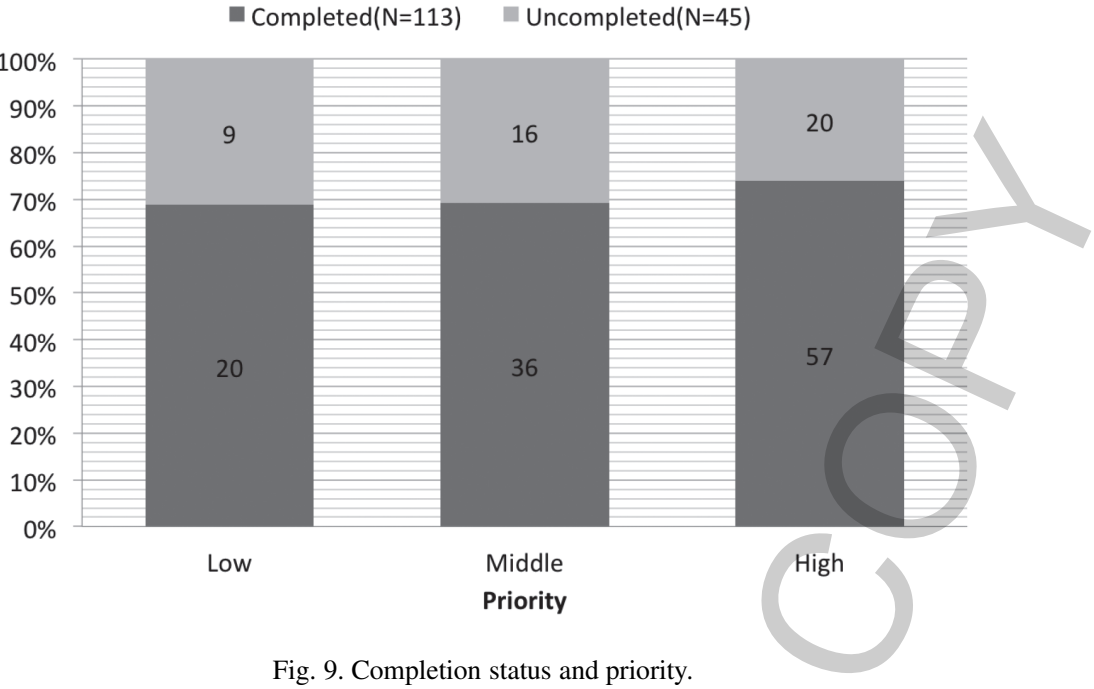

Using the above formula, we get 100 if a task took twice as long as was estimated (i.e., $100 \%$ more), and -50 if a task took half the estimated time (i.e., $50 \%$ less).

The results, using 112 completed tasks (one was not reported), are shown in Table 1 . As can be seen, in $38 \%$ of tasks, estimated time and actual time were the same. The median estimated time for such tasks was 120 minutes (IQR: 120). A similar proportion (39\%) of tasks took less actual task completion time than estimated. The mean value of the gap was -50 (IQR: 16.7) which means half of what was estimated. Finally, $22 \%$ of tasks took more time that estimated, and the median value of the gap was 100 (IQR: 100) which means twice what was estimated.

Overall, over $60 \%$ of tasks had a gap between actual and estimated times. This suggests that students often found it difficult to estimate accurately how long a given task might take. However, this does not seem to correlate with the length of estimated time (i.e., inferred task size).

For reference, we repeated the same analysis on 44 uncompleted tasks (one was not valid), and the results are shown in Table 2. Given that these are uncompleted tasks, students are likely to need more time to complete. Then, at least 15 tasks (34\%) are likely to take more time to complete than estimated. This means that uncompleted tasks are more likely to take longer than estimated, as compared to completed tasks. 
Table 2

Gap between estimated and actual time of uncompleted tasks

\begin{tabular}{cc}
\hline & Frequency $(N=44)$ \\
\hline Gap $>0$ & $9(20.45 \%)$ \\
Gap $=0$ & $6(13.64 \%)$ \\
Gap $<0$ & $29(65.90 \%)$ \\
\hline
\end{tabular}

- Completed( $\mathrm{N}=113)$ Uncompleted( $\mathrm{N}=44)$

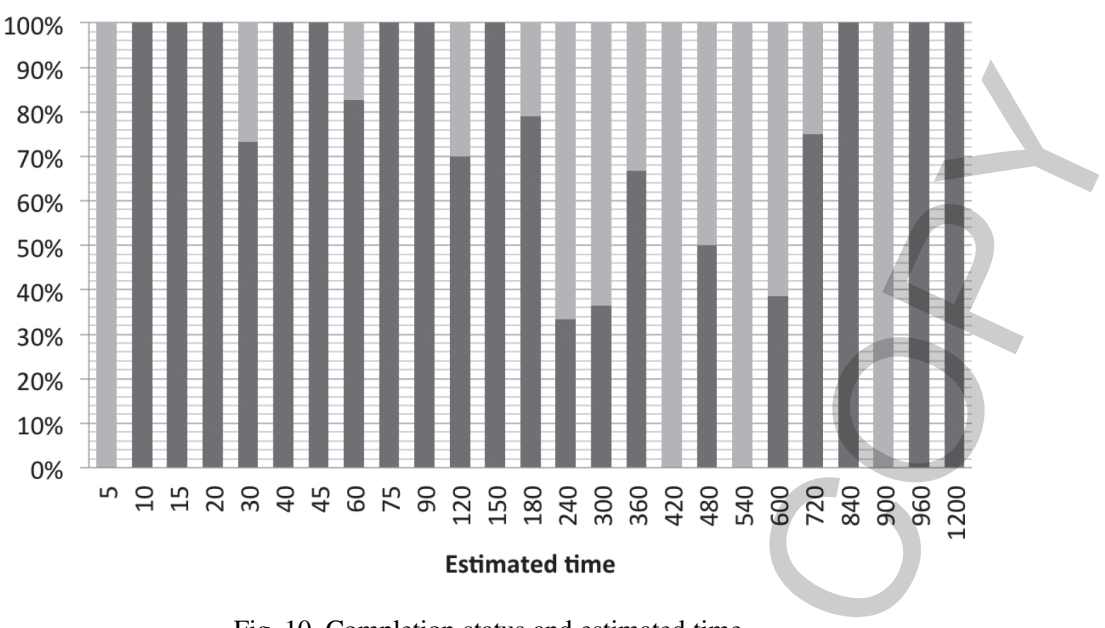

Fig. 10. Completion status and estimated time.

The next analysis looked at the relationship between the estimated time and completion status, and the results are shown in Fig. 10. As can be seen, tasks with an estimated time of between 5 and 180 minutes had a higher level of completion, while longer tasks had a higher level of non-completion across the time range. The median estimated time of completed tasks was $90 \mathrm{~min}$ (IQR: 137.5), while it was $300 \mathrm{~min}$ (IQR: 450) for uncompleted tasks. Therefore, there is a big difference between completed and uncompleted tasks as regards their estimated time.

Finally, we had a closer look at the self-reported reasons for uncompleted and nonstarted tasks, and the results are shown in Table 3 . The first factor we notice is time management, and this seems closely related to the ability to estimate task completion time (Reason 1). As we saw earlier, not all the anticipated tasks proved necessary in the end (Reason 2, 6 and 9). They were related to priority judgment.

Another set of factors is related to coordination with others (Reason 4, 7, 8). Lack of coordination with fellow collaborators, preparation for the task, and dependency on other tasks seems to be one of the major factors for uncompleted and non-started tasks. More importantly, this result suggests that some university students might not be aware of the importance of coordination for the completion of multiple tasks. 
Table 3

Reasons for non-started tasks

\begin{tabular}{clc}
\hline & Reason & \\
\hline 1 & Lack of time & $24(26.97 \%)$ \\
2 & Left it until later because of low priority & $14(15.73 \%)$ \\
3 & Forgot & $11(12.36 \%)$ \\
4 & Coordination with others didn't go smoothly & $10(11.24 \%)$ \\
5 & Because task was tiresome & $8(8.99 \%)$ \\
6 & Realized that the task was not necessary & $3(3.37 \%)$ \\
7 & Some tools were not available & $2(2.25 \%)$ \\
8 & Previous (dependent) task wasn't completed & $2(2.25 \%)$ \\
9 & New tasks with a higher priority were added & $2(2.25 \%)$ \\
12 & Lack of money & $1(1.12 \%)$ \\
13 & Other & $6(6.74 \%)$ \\
& No answer & $6(6.74 \%)$ \\
\hline
\end{tabular}

\subsection{Task grouping}

Categorizing tasks is an important part of task management. Prioritizing tasks can be seen as a form of task categorization. To understand how participants perform categorization of tasks, we asked them to group their tasks and to label the groups in the first week. The criteria for grouping tasks were left entirely up to the participants; we did not make any suggestions.

Table 4 shows participants' grouping methods along with the proportion of started, non-started, completed, and uncompleted tasks. Out of 24 participants, 17 grouped tasks based on themes (or topics) such as "about classes, projects, job hunting, hobbies" or "reports, cooking, socializing". On the other hand, 4 participants grouped tasks based on priorities such as "never forget, ASAP, When have time" or "strict deadline, weak deadline, no deadline". One participant grouped tasks based on their estimated length, such as "future plan, long-term plan, routine, ASAP". A couple of participants mixed some of these grouping criteria.

As for the relationship between the grouping methods and the proportion of various progress statuses, no obvious pattern was observed.

These results suggest at least two points. One is that grouping tasks based on theme seems to be intuitive to university students, and it could be the case that other grouping criteria rarely come to their mind. Another is that more systematic investigation is needed to understand the relationship between the grouping methods and their completion rate.

\section{Discussion}

This study aimed to understand the practice and experience of task management by university students by surveying one week's planning of their activities. This section summarizes the main findings of our investigation and discusses their implications for the design of task management support. 
Table 4

Task grouping methods and task progress

\begin{tabular}{ccccccc}
\hline ID & Grouping method & No of tasks & Non-started $(\%)$ & Started $(\%)$ & Completed $(\%)$ & Uncompleted $(\%)$ \\
\hline 1 & Priority & 5 & 20.0 & 80.0 & 75.0 & 25.0 \\
2 & Topic & 9 & 44.4 & 55.6 & 60.0 & 40.0 \\
3 & Topic & 9 & 11.1 & 88.9 & 87.5 & 12.5 \\
4 & Long-term task, & 7 & 0.0 & 100.0 & 57.1 & 42.9 \\
& habit, urgent task & & & & & \\
5 & Topic & 10 & 0.0 & 100.0 & 50.0 & 50.0 \\
6 & Topic & 6 & 16.7 & 83.3 & 60.0 & 40.0 \\
7 & Topic & 5 & 40.0 & 60.0 & 66.7 & 33.3 \\
8 & Topic & 14 & 14.3 & 85.7 & 83.3 & 16.7 \\
9 & Topic & 14 & 21.4 & 78.6 & 54.5 & 45.5 \\
10 & Topic & 14 & 35.7 & 64.3 & 44.4 & 55.6 \\
11 & Topic & 14 & 14.3 & 85.7 & 83.3 & 16.7 \\
12 & Topic & 4 & 50.0 & 50.0 & 100.0 & 0.0 \\
13 & Topic & 6 & 0.0 & 100.0 & 83.3 & 16.7 \\
14 & Topic & 12 & 41.7 & 58.3 & 100.0 & 0.0 \\
15 & Topic & 2 & 0.0 & 100.0 & 0.0 & 100.0 \\
16 & Priority & 6 & 33.3 & 66.7 & 75.0 & 25.0 \\
17 & Priority & 12 & 25.0 & 75.0 & 88.9 & 11.1 \\
18 & Topic & 3 & 0.0 & 100.0 & 33.3 & 66.7 \\
19 & Topic + Priority & 11 & 9.1 & 90.9 & 80.0 & 20.0 \\
20 & Topic & 11 & 45.5 & 54.5 & 83.3 & 16.7 \\
21 & [Missing] & 8 & 12.5 & 87.5 & 85.7 & 14.3 \\
22 & Topic & 7 & 14.3 & 85.7 & 66.7 & 33.3 \\
23 & Topic & 5 & 0.0 & 100.0 & 80.0 & 20.0 \\
24 & Priority & 8 & 37.5 & 62.5 & 60.0 & 40.0 \\
\hline
\end{tabular}

\subsection{Main findings and implications}

First, overall, $21.8 \%$ of tasks were not even started, and $22.3 \%$ of tasks were started but not completed. Therefore, nearly $44 \%$ of tasks did not progress according to the estimate. This suggests that university students do need support in their task management. Second, as can be imagined, university students must engage with various kind of tasks at busy times of year. Study related tasks formed the majority, but were still only about $40 \%$. Therefore, supporting only study-related tasks might not have significant impact on overall task management. A positive aspect of their practice is that students were more likely to start those tasks with a high priority than those with a low priority.

The task size seems to be problematic. Many participants seemed to perceive tasks that could be accomplished in few hours. It was the large tasks which were more likely to suffer from coordination problems. Therefore, one area that needs particular support is the ability to divide a task into more manageable chunks. The gap analysis also suggests that students' estimation of the time needed to complete a task can be inaccurate. A related common factor for not completing a task on time was coordination. University students need to engage with more complex and larger-scale tasks than they have before, but they may not have enough experience to coordinate with collaborators, other dependent tasks, and tools. 
Most students in our study grouped their tasks based on themes, and some grouped based on priority. Furthermore, the degree of task progress varied a lot among those grouped by themes. This suggests that there is room for investigating the effect of other types of task grouping on the progress of tasks. The literature of task management for professionals suggests grouping criteria such as deadline, location, role in a group, and collaborator [212]. Since these criteria do not seem to occur to students intuitively, it is worth investigating how they perceive such new ways of task grouping.

Overall, some of the behavior they exhibited was shared with that of professionals. In this sense, the task management tools developed for professionals might be equally effective for university students. However, further investigation is needed to measure the performance of such techniques and tools, and we plan to do that.

\subsection{Limitations}

Finally, it should be noted that this study has several limitations. First, we had a limited number of participants from a single university in our experiment, although their academic background varied and the sample size was in line with a previous study (e.g. [5]). Second, the season of the experiment was also focused on a particularly busy period due to the nature of study. It is possible that students' task management behavior would be different in a quieter period. Third, the range of tasks identified during our study should be valid for university students in many countries, but the effect of cultural differences on task diversity was not examined in this study. Therefore, our findings should not be extended to a different context, and further studies are needed to gain a comprehensive understanding of the task management behavior of university students and its support.

\section{Conclusion}

This paper reported the results of a survey that investigated the practice and experience of task management by university students. A total of 202 tasks reported by 24 university students were analyzed to survey the practice and experience of their task management. The results suggest that the university students in our survey had a reasonable sense of priority of tasks, that they tended to perceive a task as a big chunk, not a series of small chunks, that estimated time can be a good indicator of task completion, that time management and loss of coordination are the main factors for uncompleted or non-started tasks, and finally that their notion of task grouping was dominated by task themes or topics. These findings informed us as to several ways in which university students could be supported in the management of multiple tasks. 


\section{References}

[1] E. Agichtein, R.W. White, S.T. Dumais and P.N. Bennett, Search, Interrupted: Understanding and Predicting Search Task Continuation. Proceeding SIGIR '12 Proceedings of the 35th international ACM SIGIR conference on Research and development in information retrieval, 2012, pp. 315-324.

[2] V. Bellotti, B. Dalal, N. Good, P. Flynn and D.G. Bobrow. What a to do: studies of task management towards the design of a personal task list manager, Proceedings of the SIGCHI Conference on Human Factors in Computing Systems, 2004, pp. 735-742.

[3] D. Coviello, A. Ichino and N. Persico, Time allocation and task juggling, The American Economic Review 104(2) (2014), 609-623.

[4] M. Czerwinski, E. Horvitz and S. Wilhite, A diary study of task switching and interruptions, Proceeding CHI '04 Proceedings of the SIGCHI Conference on Human Factors in Computing Systems, 2004, pp. 175-182.

[5] M. Haraty, D. Tam, S. Haddad, J. McGrenere and C. Tang, Individual Differences in Personal Task Management: A Field Study in an Academic Setting, Proceedings of Graphics Interface 2012, 2012, pp. 35-44.

[6] R. Junco and S.R. Cotton, Perceived academic effects of instant messaging use, Computers \& Education 56(2) (2011), 370-378.

[7] D.M. Levy, J.O. Wobbrock, A.W. Kaszniak and M. Ostergren, Initial Results from a Study of the Effects of Meditation on Multitasking Performance, Proceedings of the 2011 annual conference on Human factors in computing systems, 2011, pp. 2011-2016.

[8] S.-J. Lin and N. Belkin, Validation of a Model of Information Seeking Over Multitasking Search Sessions, Journal of American Society for Information Science and Technology 56(4) (2005), 393415.

[9] H.L. O'Brien and E.G. Toms, What is User Engagement? A Conceptual Framework for Defining User Engagement with Technology, Journal of the American Society for Information Science and Technology 59(6) (2008), 938-955.

[10] A. Spink, H.C. Ozmutlu and S. Ozmutlu, Multitasking Information Seeking and Searching Processes, Journal of the American Society for Information Sicence and Technology 53(8) (2002), 639-652.

[11] A. Spink, M. Park, B.J. Jansen and J. Pedersen. Multitasking during Web Search Session, Information Processing and Management 42(1) (2006), 264-275.

[12] P.J. Wild, P. Johnson and H. Johnson, Understanding Task Grouping Strategies, Proceedings of the HCI 2003 Conference on People and Computers XVII, 2003, pp. 3-20. 